
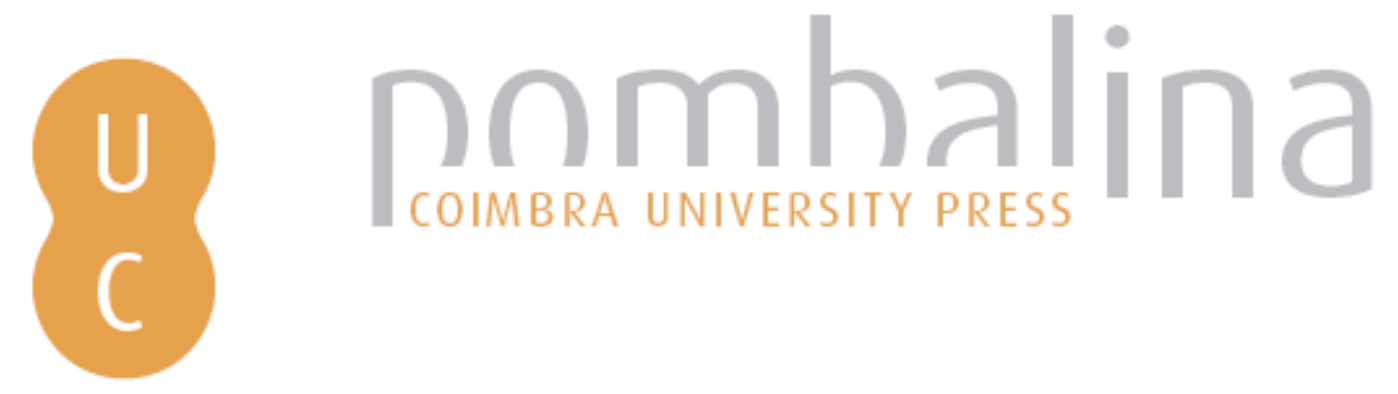

La alfabetización digital, un nuevo desafío en la Educación de Jóvenes y Adultos: un análisis freireano de las políticas de integración de las tecnologías en Uruguay

Autor(es): $\quad$ Martínez Barcellos, Rosana; Leite, Carlinda; Monteiro, Angélica María

Publicado por: Imprensa da Universidade de Coimbra

URL

persistente: URI:http://hdl.handle.net/10316.2/41316

DOI: DOI:https://doi.org/10.14195/978 $\quad$ D89 26 1326-0_27

Accessed : $\quad$ 26-Apr-2023 15:44:41

A navegação consulta e descarregamento dos títulos inseridos nas Bibliotecas Digitais UC Digitalis, UC Pombalina e UC Impactum, pressupõem a aceitação plena e sem reservas dos Termos e Condições de Uso destas Bibliotecas Digitais, disponíveis em https://digitalis.uc.pt/pt-pt/termos.

Conforme exposto nos referidos Termos e Condições de Uso, o descarregamento de títulos de acesso restrito requer uma licença válida de autorização devendo o utilizador aceder ao(s) documento(s) a partir de um endereço de IP da instituição detentora da supramencionada licença.

Ao utilizador é apenas permitido o descarregamento para uso pessoal, pelo que o emprego do(s) título(s) descarregado(s) para outro fim, designadamente comercial, carece de autorização do respetivo autor ou editor da obra.

Na medida em que todas as obras da UC Digitalis se encontram protegidas pelo Código do Direito de Autor e Direitos Conexos e demais legislação aplicável, toda a cópia, parcial ou total, deste documento, nos casos em que é legalmente admitida, deverá conter ou fazer-se acompanhar por este aviso.

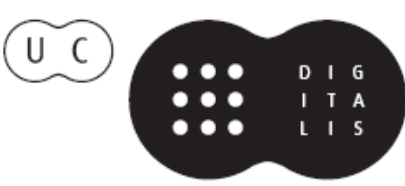




\title{
27. LA ALFABETIZACIÓN DIGITAL, UN NUEVO DESAFÍO EN LA EDUCACIÓN DE JÓVENES YADULTOS: UN ANÁLISIS FREIREANO DE LAS POLÍTICAS DE INTEGRACIÓN DE LAS TECNOLOGÍAS ENURUGUAY
}

\author{
Rosana Martínez Barcellos ${ }^{132}$ \\ Carlinda Leite 133 \\ Angélica María Monteiro ${ }^{134}$
}

\begin{abstract}
Resumo
Este artigo analisa as políticas de integração das tecnologias do Uruguai em seu contexto regional, e a importância da alfabetização digital no segmento da educação de adultos, visando o seu desenvolvimento pleno na sociedade onde as relações da tecnologia, cultura, e identidade estruturam a vida (Castells, 1999; Kellner \& Share, 2008). Para sustentar esta análise, nos propomos desde uma conceção de alfabetização no sentido amplo como propôs Paulo Freire (1987), que permita conhecer e transformar a realidade, e o conceito de educação dialógica,
\end{abstract}

132 Faculdade de Psicologia e de Ciências da Educação. Universidade do Porto.

133 CIIE. Faculdade de Psicologia e de Ciências da Educação. Universidade do Porto.

134 CIIE. FCT. Faculdade de Psicologia e de Ciências da Educação. Universidade do Porto. 
libertadora (Freire, 1987), que compreende a educação como processo de emancipação.

No Uruguai, desde a saída da ditadura e com maior ênfase desde o ano de 2007, o primeiro governo de esquerda colocara em prática diversas políticas de integração com as tecnologias. No contexto da Agenda digital Uruguaia, têm-se desenvolvido diversas estratégias na educação que têm possibilitado, às gerações adultas do interior do país, participar da alfabetização digital, com o objetivo de diminuir a brecha digital e promovendo a participação e a integração social.

Uma análise destas políticas de alfabetização digital para adultos permite concluir que elas são elementos-chave no desenvolvimento da sociedade mais democrática, livres e inclusivas, e constituem, em si mesmas, um desafio para os governos. Seguindo a linha de pensamento freireana, os indivíduos necessitam ferramentas que lhes permitam realizar uma leitura da realidade de forma crítica, levando em conta as possibilidades que as tecnologias digitais têm introduzido e as formas de comunicação que têm imposto.

\footnotetext{
Abstract

This paper analyzes Uruguay's technologies integration policies, in its regional context, and, also, states the importance of digital literacy in youth and adult education, taking into account a conception of full development of individuals in society, where relationships between technology, culture and identity structure life (Castells, 1999; Kellner \& Share, 2008). To support this analysis, we start from a broad sense of literacy, as proposed by Paulo Freire (1987), a literacy which enables individuals to understand and transform reality. Besides, we also start from concepts of dialogical education
} 
and education for freedom, which comprise education as an emancipation process.

In Uruguay, since the exit of the dictatorship and with greater emphasis since 2007, the first left-wing government had put into practice several integration policies regarding technologies. In the context of the Digital Agenda of Uruguay, several educative strategies have been developed. Such strategies have been allowed adult generations to participate in digital literacy, reducing the digital gap and promoting participation and social integration. An analysis of such digital literacy policies for adults shows that they are key elements in the development of a more democratic, free and inclusive society. Such policies constitute, themselves, challenges for governments. Following the line of Freire's thinking, individuals need tools that allow them to develop a critical reading of reality, taking into account the possibilities introduced by digital technologies and the patterns of communication that have been promoted in this context. 
Este siglo XXI ha recibido como legado las transformaciones sociales, económicas, políticas, científicas y educativas que el siglo anterior se encargó de producir a gran velocidad. En América Latina, el inicio de este siglo fue marcado por un viraje en la dirigencia de los gobiernos y por lo tanto, un cambio muy importante en las políticas sociales. En muchos países de este continente pasaron a ser comandados por gobiernos de izquierda. Conjuntamente con los cambios políticos vinieron aparejadas una serie de mudanzas que trajeron esperanza para la integración de una sociedad fragmentada por las diferencias.

Esas dinámicas sociales, se vieron estructuradas por un contexto definido por las características de una sociedad pos-industrial fuertemente influenciado por el desarrollo de las tecnologías de la información y la comunicación, donde la forma de producción y circulación del conocimiento establecen nuevas relaciones entre los sujetos. Relaciones que pasan por diversos aspectos de la vida cotidiana: aspectos laborales, económicos, del ejercicio de la ciudadanía, comunicacionales etc. Surgen, pues, con los avances tecnológicos muchas oportunidades, pero también nuevas formas de exclusión social, determinadas no sólo por el acceso material a los dispositivos sino también por la posibilidad de hacer uso efectivo de las tecnologías digitales.

Para enfrentar este reto, las políticas en educación de adultos tienen un nuevo desafío, la alfabetización digital. La que permita al sujeto aprehender esta realidad, cada vez más dependiente y determinada por las tecnologías digitales, participando activamente en ella. Una alfabetización que apunte a lo que plantea Coll (2009):

El concepto de alfabetización (...) comporta algo más que el conocimiento y manejo de unos recursos simbólicos y unas tecnologías, sea cual sea la naturaleza y características de esos recursos y esas tecnologías. Comporta además conocer las prácticas socioculturales 
asociadas al manejo de los recursos simbólicos y las tecnologías en cuestión y ser capaz de participar en dichas prácticas utilizando unos y otras de manera adecuada. (Coll, 2009, p. 125)

Esta idea de cierto modo, se aproxima a la sustentada por Freire (1978), en la que resalta la necesidad de aprender a leer el mundo. $\mathrm{Y}$ es en ese acto de leer en el que se pone en juego la capacidad crítica que permite comprender y dar significado a la palabra en relación al contexto, al mundo de cada individuo.

Alfabetización crítica implica lectura crítica, decía Freire (2005, p. 11), "que não se esgota na decodificação pura da palavra escrita ou da linguagem escrita, mas que se antecipa e se alonga na inteligência do mundo”. Esta lectura crítica es la clave principal para el desarrollo de una alfabetización digital que se encuadre en la realidad, y que atienda a las verdaderas necesidades de los educandos.

Por lo tanto, la alfabetización en el siglo XXI va más allá de aprehender la palabra. Hoy, los individuos necesitan mucho más que manejar el código escrito, necesitan herramientas que les permitan interactuar en un mundo en constante cambio, de lo contrario son excluidos de innúmeras formas de ejercicio de sus derechos. El enorme desarrollo de las tecnologías digitales ha determinado no sólo nuevos sentidos y significados, como lo plantean Sartori e Soares (2005), con éstas nuevas formas de fragmentación social. Existe una brecha entre los que pueden acceder a las tecnologías digitales y los que no lo tienen, y entre los que además de tener acceso material, pueden hacer uso efectivo de sus potencialidades en el desarrollo de la vida cotidiana y los que permanecen ajenos a ellas. Como lo realzó Streck (2009) al analizar la Pedagogía del Oprimido (Freire, 1987), los individuos son seres históricos y por lo tanto, las exigencias de cada época constituyen parámetros de inclusión/exclusión particulares. Hoy, el modo de usar las tecnologías digitales es uno de esos parámetros y merece atención. 
Es así que iniciamos este artículo realizando un breve recorrido por las políticas de integración de tecnologías en Uruguay y su encuadramiento en las políticas que tienen un alcance regional e internacional. A partir de ello, tejemos una serie de ideas que apuntan a la importancia de la alfabetización digital en adultos y la forma que ha sido abordada en Uruguay. Para ello, presentamos el Plan Nacional de Alfabetización Digital (PNAD) como ejemplo de política de alfabetización de adultos en un contexto digital y como política de integración social. Realizamos un análisis de la relación entre alfabetización digital y ejercicio de la ciudadanía, tejiendo relaciones con concepciones que giran en torno a una educación liberadora, emancipadora, propuesta por Paulo Freire (1987). Finalizamos este artículo con algunas conclusiones respecto a la importancia de la alfabetización digital en adultos y los desafíos que ésta presenta para las políticas en educación. Concluimos que estas políticas son un elemento clave en el desarrollo de sociedades más democráticas, libres e inclusivas, y constituyen, en sí mismas, un desafío para los gobiernos. Siguiendo la línea de pensamiento freireana, los individuos necesitan herramientas que les permitan realizar una lectura de realidad de forma crítica, teniendo en cuenta las posibilidades que las tecnologías digitales han introducido y las formas de comunicación que han impuesto.

\section{Un contexto político para las Tecnologías Digitales}

En materia de políticas, el siglo XXI se ha caracterizado por el desarrollo de agendas educativas y sociales que tienen como centro la inclusión de las tecnologías de la información y comunicación en los diferentes sectores. A nivel mundial y regional, las tendencias apuntan a un fortalecimiento de esas políticas, lo que podemos observar desde el año 2001 con la aprobación 
de la Asamblea de las Naciones Unidas a la realización de la Cumbre Mundial de la Sociedad de la Información (CMSI) que se llevó a cabo en Ginebra en el año 2003. Como resultado de esta, surgieron acuerdos internacionales que pusieron énfasis en la integración de las TIC fuertemente relacionada al desarrollo económico y social de los países, y las exigencias de la sociedad de conocimiento y la información.

A nivel latinoamericano, la XVIII Cumbre Iberoamericana de Jefes de Estado y de Gobierno, llevada a cabo en El Salvador en el año 2008, resalta la importancia de enfocar fuerzas en las políticas que garanticen la equidad, justicia social, participación e inclusión. En el acuerdo surgido de esta Cumbre (Gobierno", 2008), los representantes de los veintidós países integrantes de la Comunidad Iberoamericana, se "comprometen a mejorar las oportunidades reales de las y los jóvenes, que permitan su crecimiento integral para lograr mayores niveles de inclusión y desarrollo social..." (Declaración de San Salvador, 2008, p. 2).

Como producto de esta Cumbre Iberoamericana, se impulsa el compromiso colectivo de los países de esta zona geográfica en el Proyecto Metas 2021. De él resaltamos la meta general, "Ofrecer a todas las personas oportunidades de educación a lo largo de toda la vida" (CEPAL \& OEI, 2010, p. 156), que busca de forma específica garantizar el acceso a la educación a personas en situación más desfavorecida y aumentar la partición de estos adultos y jóvenes en programas de formación continua presenciales y a distancia.

En el mismo sentido de estas políticas, que promueven la educación a lo largo de la vida, el desarrollo de capacidades, y el enriquecimiento de los conocimientos (principios que integran la Declaración de Hamburgo de 1977 y en los que se basan las políticas de educación de adultos de las naciones integrantes de la ONU), la ONU, a través de la Conferencia Internacional de Educación de Adultos (CONFINTEA VI), consolida esta idea planteando que: 
Afirmamos que a alfabetização é o alicerce mais importante sobre o qual se devem construir aprendizagens abrangentes, inclusivas e integradas ao longo de toda a vida para todos os jovens e adultos. Diante da dimensão do desafio da alfabetização global, consideramos fundamental redobrar nossos esforços para garantir que as prioridades e os objetivos de alfabetização de adulto já existentes, conforme consagrados na Educação para Todos (EPT), na Década das Nações Unidas para a Alfabetização (United Nations Literacy Decade, em inglês) e na Iniciativa de Alfabetização para o Empoderamento (Literacy Initiative for Empowerment, em inglês), sejam alcançados por todos os meios possíveis. (UNESCO, 2010, p. 6)

Ese empoderamiento, resultado de una educación para, y a través de la vida, como derecho humano fundamental, es esencial para que hombres y mujeres accedan a otros derechos. En la Declaración de Montevideo (MERCOSUR, 2009), los Ministros de Educación del MERCOSUR, resaltaron el carácter de bien público que posee la educación, y como tal, deben ser asegurados los medios para que adultos y jóvenes accedan a ella con el fin de contribuir a la formación de hombres y mujeres "reflexivos y autónomos, capaces de protagonizar la justicia, la solidaridad, la libertad, la democracia, la integración social, la defensa del ambiente, la convivencia pacífica y la no discriminación." (MEC, 2011, p. 14). Los medios para alcanzar esto, deben contemplar las necesidades individuales, las características propias de cada cultura y cada sociedad, de tal forma que se pueda dar la apropiación de conocimientos, capacidades, y herramientas que permitan el desarrollo pleno en la sociedad.

En este proceso de construcción de sociedades más inclusivas y de equidad social, en el que se han embarcado los países latinoamericanos, la alfabetización digital es un puntal esencial para el 
ejercicio de los derechos de los individuos, y por lo tanto, el ejercicio de la ciudadanía. Esta preocupación es evidente en las Metas 2021, donde refiere que: "Actualmente, la inclusión social se vincula, cada vez más, con el acceso al conocimiento, por la participación en redes y por el uso de las TIC" (CEPAL \& OEI, 2010, p. 72).

Por lo tanto, la alfabetización digital, debe ser pensada desde esta perspectiva, comprendiendo que los cambios que las tecnologías digitales han venido a imponer, generan relaciones "opresor"-"oprimido", y tienen como resultado nuevas formas y parámetros de inclusión/exclusión en la sociedad del conocimiento. La alfabetización digital, debe ser considerada un derecho, en el marco de una educación liberadora, en el sentido que defendía Freire (Freire, 1978). Educación que se centra en la formación de seres reflexivos, autónomos, solidarios, justos, con una sólida conciencia democrática que los empuje a luchar por la integración social.

Uruguay abrazó plenamente esta idea y con el primer gobierno de izquierda (2005-2010) se inició un proceso de transformación que tuvo como protagonista principal a la Educación y las TIC, a través de políticas de democratización y acceso a internet y acortamiento de la brecha digital existente entre diferentes sectores de la sociedad. Es así que en el año 2007 se inició lo que se conoce como "Plan Ceibal" (Decreto 144/007), un plan político que se transformó en Proyecto Pedagógico, inspirado en el modelo One Laptop Per Child (OLPC) del Instituto Tecnológico de Massachusetts (MIT).

Estas transformaciones impulsaron entre otras cosas: la universalización del acceso a las tecnologías de la información y comunicación en la Educación Pública, principalmente en Educación Básica. Pero el fenómeno Ceibal salió rápidamente del aula para ser parte de toda la sociedad. Los padres, abuelos, vecinos comenzaron a tener curiosidad por el mundo al que podían acceder a través de las pequeñas pantallas de las computadoras. 
Traemos a memoria una de las entrevistas realizadas a una de las primeras docentes que recibieron las computadoras ${ }^{135}$, en la que nos relataba como los padres comenzaron a ser enseñados por sus hijos para poder usar las computadoras y realizar el seguimiento de su producción de leche en el tambo familiar, que era enviada a la Cooperativa a la que pertenecían. Este simple hecho les permitía conocer con certeza la cantidad de leche producida y lo que significaba en términos monetarios. Como este ejemplo podemos mencionar muchos otros que quedaron marcados en nuestra sociedad y que seguramente fueron los motores para que iniciativas de alfabetización digital de adultos se desarrollaran en todo el país, concretizadas en el Plan Nacional de Alfabetización Digital (PNAD) coordinado por el Ministerio de Educación y Cultura al que se le asociaron otros actores estatales y no estatales.

Los fundamentos del PNAD, tiene sus bases en la idea que venimos desarrollando, donde se valoriza la importancia de la alfabetización digital para la inclusión social. Entre sus objetivos generales, podemos observar que en cierta medida, corren por el mismo carril que los conceptos de alfabetización, de lectura del mundo, de educación liberadora, de Paulo Freire. Encontramos pues, que estos objetivos visan por asegurar el acceso a las herramientas que permitan la lectura de un mundo cuyas relaciones y funcionamiento está sometido cada vez más a las exigencias derivadas de los desarrollos tecnológicos, y para la participación activa y crítica en él. A través del PNAD se busca:

Promover la alfabetización digital de adultos como forma de disminuir la brecha digital generacional, impulsando la utilización

135 Las laptops entregadas por el Plan Ceibal son totalmente gratuitas. Su modelo es el propuesto por OLPC, y son conocidas como XO o ceibalitas. 
de las TIC como medio para mejorar y acrecentar el acceso a bienes y servicios educativos y culturales, contribuyendo a través de ello a la integración social.

Contribuir a la cohesión social a través de una inclusión digital que fomente la integración de las personas como sujetos críticos y activos, trascendiendo el rol de consumidores de tecnologías y contenidos digitales para ser creadores, de acuerdo a los distintos niveles de apropiación que cada uno incorpore.

Cooperar con las políticas de desarrollo de gobierno electrónico del Estado a través del diseño e implementación de talleres temáticos orientados a "educar la demanda", de forma de democratizar las informaciones y la utilización de los servicios disponibles. (MEC, 2010, p. 4)

Estos objetivos se acompañan de una serie de estrategias que se desarrollan a distintos ámbitos educativos: formal y no formal. En los que se estructuran diversas actividades de participación ciudadana, concepto que veremos en el capítulo siguiente y que se ha resignificado a partir de la construcción de un nuevo tipo de ciudadanía, en la que las TIC ocupan un lugar fundamental, donde "el alfabetizando... se insere num processo criador, de que ele é também sujeito" (Freire, 2005, p. 29).

De acuerdo a lo expuesto anteriormente, podemos decir que en Uruguay, se han desarrollado desde el año 2007, una serie de políticas que visan por el desarrollo de más infraestructura tecnológica, más integración de tecnologías digitales a nivel de gobierno, educación y promoción de cultura. Sin embargo, ya desde el inicio de estas políticas, se vio la necesidad de reforzar un aspecto fundamental, para que no fueran en contra de las políticas de inclusión social. Esta necesidad surgió de las demandas que existían en la población adulta, a partir de las exigencias y oportunidades de las incorporaciones tecnológicas en la socie- 
dad $^{136}$. Especialmente, entre los sectores de la población más vulnerabilizados.

En respuesta a esta demanda, el Ministerio de Educación y Cultura (MEC), se encarga de llevar adelante una política centralizada, que tiene como fin, la formación de ciudadanos "más aptos para vivir en una sociedad en la que el conocimiento, la capacidad de compartir y la facilidad para incorporar nuevas aptitudes son la clave para la actividad laboral, académica o simplemente para la relación con los otros" (Elissalde, Acosta, \& Recalde, 2008, p. 4). Establece así, los Centros MEC, ubicados en el interior del país, con docentes que pertenecen a las propias comunidades y conocen la realidad del entorno. Uno de los pilares fundamentales de estos Centros, es la alfabetización digital, y tiene como objetivo "facilitar el acceso a bienes y servicios culturales a poblaciones que tradicionalmente han quedado marginadas de las rutas de circulación de dichos bienes" (MEC, 2010, p. 9). Al mismo tiempo, orienta todas sus actividades a lograr una "la cohesión social en poblaciones con menos de cinco mil habitantes" (ídem).

De esta política queremos resaltar la importancia que se le dio a "educar la demanda" (Elissalde et al., 2008), fortaleciendo los puntos más débiles de toda esta enorme red que se despliega para concretar los objetivos de las políticas que giran en torno a la sociedad del conocimiento y la información. Esos puntos débiles son representados por la falta de políticas orientadas a una educación que permita la inclusión social, que genere puentes para romper con las brechas sociales, generacionales y económicas que separan a los individuos de la posibilidad de participar de la vida social de este siglo. Los Centros MEC se proponen brindar

136 Así es planteado en el documento presentado por el MEC en el que se presentan las líneas de acción de los Centros MEC, encargados de impulsar políticas de alfabetización digital de adultos en el interior del país. 
conocimientos para que los individuos puedan intervenir en la realidad, idea que sigue la misma línea de Freire (1996), cuando plantea la necesidad de ser un sujeto partícipe de la historia, de constatar (en el sentido de observar y conocer) "no para me adaptar mas para mudar" (Op. cit., p. 77).

Pensar en un individuo que participa en su historia, que la conoce, que la cambia, es pensar en un individuo libre, autónomo, que su acción impacta sobre el mundo y a la vez le da significado. En una sociedad que cambia, con problemas viejos en contextos nuevos, los individuos necesitan de una educación que los empodere, que los libere. Es por eso que todo esfuerzo de los pueblos en avanzar en la alfabetización digital, será un paso más para construir una sociedad más igualitaria.

Reorganizar a sociedade velha, transformá-la para criar a nova sociedade não é tão fácil assim. Por isso, não se cria a sociedade nova da noite para o dia, nem a sociedade nova aparece por acaso. A nova sociedade vai surgindo com as transformações profundas que a velha sociedade vai sofrendo. (Freire, 2005, pp. 74-75)

Las resistencias que aún existen, de los adultos hacia las tecnologías, hacen aún más desafiante la tarea. La importancia de acercar a adultos y jóvenes a las TIC, implica un acto de humildad, donde la mayor convicción debe ser: "Nadie ignora todo. Nadie sabe todo. Todos nós ignoramos alguma coisa." (Freire, 2005, p. 69). Es justamente en este sentido que los Centros MEC, se proponen romper con una serie de barreras entre los adultos y las tecnologías, entre las que plantea: fortalecer la confianza para vencer el miedo; desarrollar la capacidad crítica para valorar la información y construir su propia opinión; dotar de herramientas básicas que permitan usufruir. 
Con esta reflexión abrimos un nuevo capítulo en el que nos centramos en la importancia de la alfabetización digital para el ejercicio de la ciudadanía en un contexto en el que las TIC han modificado las estructuras sociales.

\section{La alfabetización digital y ciudadanía}

Ya en este artículo referíamos a la idea de alfabetización en sentido amplio que propuso Paulo Freire. Una alfabetización que se opone a lo puramente mecánica, que promueve la toma de conciencia en, y a partir de la realidad (Freire, 1997).

Pensávamos numa alfabetização direta e realmente ligada á democratização da cultura, que fosse uma introdução a esta democratização. Numa alfabetização que, por isso mesmo, tivesse no homem, não esse paciente do processo, cuja virtude única é ter mesmo paciência para suportar o abismo entre sua experiência existencial e o conteúdo que lhe oferecem para sua aprendizagem, mas o seu sujeito (Freire, 1997, p. 104).

Este concepto que se encuadra en una concepción de educación libertadora y responsable, que "está en relación con la naturaleza humana haciéndose y rehaciéndose en la Historia”137 (Freire, 2003b, p. 90). Clarificando el sentido atribuido por Freire a la Educación libertadora, podemos referir que es aquella que permite a los individuos ultrapasar la condición de exclusión, de marginación, para tomar su lugar en el mundo con responsabilidad, y con conciencia de que es sujeto de derechos (Monteiro \& Tavares, 2011).

137 Traducción mía. 
Así pues, hablar de alfabetización digital en una perspectiva freireana nos obliga a pensar en este proceso como un proceso de diálogo, de construcción colectiva, o sea, de un proceso en que los individuos sean partícipes activos en una conversación constante entre vida cotidiana y contenidos de aprendizaje. Este procedimiento exige que los individuos sean críticos, aspectos que la alfabetización digital puede apoyar si permite un acceso más amplio a una base de información. Por eso, la alfabetización digital debe atender a cuestiones de la vida diaria, de la comunicación, de gobierno electrónico, de cultura y de todos los otros aspectos de la vida en sociedad y relacionada con cuestiones de poder.

Que a alfabetização tem que ver com a identidade individual e de classe, que ela tem que ver com a formação da cidadania, tem. É preciso, porém, sabermos, primeiro, que ela não é a alavanca de uma tal formação - ler e escrever não são suficientes para perfilar a plenitude da cidadania -, segundo, é necessário que a tomemos e a façamos como um ato político, jamais como um que fazer neutro (Freire, 2003a, p. 58)

Las tecnologías digitales, como planteábamos desde el inicio de este texto, no son, ni serán la panacea de la humanidad. No garantizan por sí mismas la participación ciudadana y la construcción de una sociedad democrática y justa (como es planteado una y otra vez en los objetivos de las agendas políticas vinculadas a la integración y desarrollo de las tecnologías de nuestros países). Para ello, se hacen necesarios elementos que nos permitan resignificar las dinámicas que se establecen en la sociedad, dinámicas marcadas por dominios del campo tecnológico que generan dilemas entre las oportunidades y amenazas que constituyen.

$\mathrm{Y}$ es en ese lugar de conflictos que radica la importancia de ir más allá en la alfabetización digital de jóvenes y adultos para 
romper con las brechas que se han generado en el acceso y uso efectivo de las tecnologías. Jenkins (2006) afirmó que existen dos tendencias opuestas en relación a las tecnologías: una que va en desmedro de la democracia, de la libertad y de la inclusión social, y otra que tiende a proporcionar nuevas oportunidades democráticas para el ejercicio de la ciudadanía. Por eso es necesario delimitar el concepto de ciudadanía para acercarnos y comprender el de ciudadanía digital. Siguiendo las ideas de Freire (2003a), ciudadanía es una condición que se otorga al ciudadano, siendo éste un individuo que ejerce sus derechos civiles y políticos en el Estado, "quer dizer, com o uso dos direitos e o direito de ter deveres de cidadão" (Freire, 2003a, p. 45).

Como la vida en sociedad nos ha revelado, los derechos y deberes del ciudadano son fuertemente delimitados por diversas pautas de gobernanza que se basan en las tecnologías digitales. Los gobiernos avanzan con propuestas de gobierno electrónico, haciendo referencia con ello a las diversas formas de manejar y brindar la información a través de bases de datos, realizar trámites, participar en las decisiones locales o nacionales, acceder a servicios y documentación entre muchas opciones que aparecen a diario. La Organización de Estados Americanos (OEA) define al gobierno electrónico como:

[...] el uso de las Tecnologías de Información y Comunicación TIC, por parte de las instituciones de gobierno, para mejorar cualitativamente los servicios de información que se ofrecen a los ciudadanos; aumentar la eficiencia y eficacia de la gestión pública e incrementar sustantivamente la transparencia del sector público y la participación ciudadana. ${ }^{138}$

138 Portal de la Secretaría de Asuntos Políticos de la OEA. http://www.oas.org/ es/sap/dgpe/guia_egov.asp 
Sin embargo, existen brechas que dividen a la sociedad entre los que pueden ejercer este nuevo tipo de ciudadanía y los que no. Estas brechas no son sólo de tipo económico, social, o geográfico, son intergeneracionales. Hay un abismo entre generaciones, como planteaba Prensky (2001), de nativos e inmigrantes digitales, los que no conocen un mundo sin las tecnologías digitales y los que intentan participar de las lógicas determinadas por ellas para poder actuar e interactuar en ese mundo al que se sienten ajenos. Es evidente que para promover políticas que tengan como eje central el ejercicio de pleno de la ciudadanía, no se pueden obviar esas brechas sociales, económicas, educativas que están latentes en nuestros pueblos.

En ese campo de tensiones es que se encuentran los desafíos para la alfabetización digital de jóvenes y adultos vale la pena recordar de nuevo a Freire (2003a) cuando apunta hacia la importancia de: "Indagar em torno dos limites da alfabetização como prática capaz de gerar nos alfabetizandos a assunção da cidadania ou não" (Ibidem, p. 45), y cuando sustenta que ese procedimiento "Implica pensar também nos obstáculos com os quais nos defrontamos na prática..." (Ibídem, p. 45)

\section{Consideraciones finales: un análisis freireana de la alfabetización digital de jóvenes y adultos en Uruguay}

El recorrido que hemos realizado en este texto, no hace más que llevarnos a reflexionar sobre la condición de los hombres y mujeres, jóvenes y adultos de nuestras sociedades actuales. Donde las tecnologías digitales han modificado las relaciones entre los sujetos, entre ellos y el Estado, entre ellos y la cultura, entre ellos y el trabajo.

En ese escenario de cambios que hemos presentado, la alfabetización en el sentido más amplio que proponía Freire (Freire, 1987), con estrecho vínculo a la democracia, viene a traer sentido a la 
búsqueda de estrategias que permitan construir nuevos caminos para la alfabetización digital. Y esa democracia "demanda estruturas democratizantes e não estruturas inibidoras da presença participativa da sociedade civil" (Freire, 2003c). Implica construir juntos, caminos que posibiliten a los jóvenes y adultos, sobre todo, los que viven en condiciones de mayor vulnerabilidad social, acceder a sus derechos, haciendo frente a las nuevas formas de exclusión social que se desarrollan solapadamente a través de las (im)posibilidades que establecieron las tecnologías.

Esas posibilidades para unos, e imposibilidades para otros, han establecido formas de poder que profundizan las diferencias entre los que tienen acceso material a las TIC y los que no, los que además hacen uso de las posibilidades que les ofrecen las TIC para interactuar en el mundo, y los que no. Es pues, en ese sector de la población, desde el año 2007, en el que se redoblaron los esfuerzos para desarrollar políticas de alfabetización digital de jóvenes y adultos en Uruguay.

Esta alfabetización parte de la idea de que, a través de la construcción del conocimiento del mundo (y entre esos conocimientos, ponemos énfasis en los digitales), los individuos podrán sobrepasar las condiciones de exclusión que vinieron a traer, las "(im)posibilidades" impuestas por las TIC.

El desafío entonces, radica en ofrecer herramientas con las que los individuos inicien la búsqueda ininterrumpida, concientes de que la velocidad de los cambios requiere ese proceso continuo de aprendizaje en un contexto histórico y social, crítico y democrático.

En suma, el análisis realizado a partir de un contexto político a nivel regional, y nacional en Uruguay, en cuanto a la alfabetización digital de jóvenes y adultos, nos permitió concluir que el desarrollo de estas políticas, es clave para que las sociedades sean más igualitarias, democráticas y libres. Se hace cada vez más necesario, que las posibilidades de acceso a las tecnologías y el desarrollo 
de competencias digitales, sean consideradas con mucha seriedad. Teniendo en cuenta, que la brecha digital profundiza la brecha social. Es por esto, que alentamos la idea de resignificar el pensamiento freireano, para construir estrategias políticas que revolucionen la sociedad, y devuelvan la esperanza a los jóvenes y adultos que han sido excluidos, e incapacitados de ejercer en plenitud sus derechos en la sociedad del conocimiento.

\section{Referencias bibliográficas}

Castells, M. (1999). La era de la información. Economía, sociedad y cultura. El poder de la identidad. Buenos Aires: Siglo XXI.

CEPAL \& OEI. (2010). Metas Educativas 2021. Madrid: OEI.

Coll, C. (2009). Aprender y enseñar con las TIC: expectativas, realidad y potencialidades. In R. Carneiro, J. C. Toscano \& T. Díaz (Eds.), Los desafíos de las TIC para el cambio educativo. Madrid: OEI. Fundación Santillana.

Elissalde, R., Acosta, K., \& Recalde, A. (2008). Más allá de la alfabetización digital. Educar la demanda. Montevideo: UNESCO/Centros MEC.

Freire, P. (1978). Pedagogía del Oprimido. Madrid: Siglo XXI.

Freire, P. (1987). Pedagogia do Oprimido (17. a ed.). Rio de Janeiro: Paz e Terra.

Freire, P. (1996). Pedagogia da autonomia: Saberes necessários à prática educativa (31. ${ }^{\text {a }}$ ed.). São Paulo: Paz e Terra.

Freire, P. (1997). Educação como prática da liberdade. Rio de Janeiro: Paz e Terra.

Freire, P. (2003a). Alfabetização como elemento de formação da cidadania. Política e Educação (7. ${ }^{\text {a }}$ ed., Vol. 23). São Paulo: Cortez Editora.

Freire, P. (2003b). Educação e responsabilidade Políticas e Educação: Ensaios (pp. 89-95). São Paulo: Cortez Editora.

Freire, P. (2003c). Politica e Educação. São Paulo: Cortez Editora.

Freire, P. (2005). A importância do ato de ler. Em três artigos que se completam (46." ed. Vol. 13). São Paulo: Cortez Editora.

Declaración de San Salvador (2008).

Jenkins, H. (2006). Convergence culture: where old and new media collide. New York: New York University.

Kellner, D. \& Share, J. (2008). Educação para a leitura crítica da mídia, democracia radical e a reconstrução da educação. Educaçã \& Sociedade, 29(104), pp. 687-715 .

MEC. (2010). Plan Nacional de Alfabetización Digital. Montevideo: MEC/Centros MEC. 
MEC. (2011). Educación de Personas Jóvenes y Adultas en el Uruguay. Experiencias actuales, desafíos y perspectivas de futuro a partir del Marco de Acción de Belém. Uruguay: MEC.

MERCOSUR. (2009). Declaración de Montevideo. In MEC (Ed.), Educación de Personas Jóvenes y Adultas en el Uruguay. Experiencias actuales, desafíos y perspectivas de futuro a partir del Marco de Acción de Belén. Montevideo: MEC

Monteiro, A. \& Tavares, C. (2011). A cidadania ativa e sua relação com a educação em direitos humanos. Revista Brasileira de Politica e Adiministração da Educação, 27(1), 13-24.

Prensky, M. (2001). Digital natives, digital immigrants. On the Horizon, 9(5), 1-6.

Sartori, A. S. \& Soares, M. P. (2005). Concepção dialógica e as NTIC: A Educomunicação e os ecossistemas comunicativos. Paper presented at the V Colóquio Internacional Paulo Freire, Recife.

Streck, D. (2009). Da pedagogia do oprimido as pedagogías da exclusão: Um breve balanço crítico. Revista Educação \& Sociedade, 30(107), 539-560.

UNESCO. (2010). Confintea VI. Brasília: UNESCO. 